\title{
Action Reflections: A Client-Centered Technique to Bridge the WHY-HOW Transition in Motivational Interviewing
}

\author{
Ken Resnicow and Fiona McMaster \\ University of Michigan, Ann Arbor, USA \\ Stephen Rollnick \\ Cardiff University, $U K$
}

\begin{abstract}
Background: When using Motivational Interviewing (MI), once resistance or ambivalence are resolved and motivation is solidified, many practitioners struggle with how best to transition the discussion toward action planning, while still retaining the spirit and style of client centeredness, i.e., moving from the WHY phase to the HOW phase of counseling in a style that is MI-consistent. For many, there is a perception that the counseling style, skills, and strategies used to build motivation are distinct from those used in the action planning phase. The WHY to HOW transition does not, however, necessitate abandoning a client-centered style for a more overtly educational or directive style. Goal setting, action planning, provision of advice, and relapse prevention can be implemented from an autonomy supportive, MI consistent framework. Method: To this end, this article will present a new class of reflection, which we have termed "action reflections", that can be used to help bridge the WHY-HOW gap. Action reflections (AR) allow the clinician to maintain a tone and orientation that are consistent with MI, i.e. autonomy support; guiding versus directing, during the action phase of counseling. They differ from reflecting change talk as they focus not on the WHY of change, but the HOW, WHEN, or WHERE. Action Reflections (ARs) also differ from the more common type of reflections such as those that focus on client feelings, rolling with resistance, or acknowledging ambivalence as ARs usually contain a potential concrete step that the client has directly or obliquely mentioned. Like any type of reflection, ARs represent the clinician's best guess for what the client has said or, more apropos here, where the conversation might be heading. Conclusion: This article describes the various types of ARs and provides examples of each to help clinicians incorporate them into their behavior change counseling.
\end{abstract}

Keywords: Motivational interviewing, skill development, new technique, WHY-HOW transition.

\section{Introduction}

Motivational Interviewing has been established as an effective technique to strengthen motivation for change across a wide range of health behaviors, particularly among resistant

Reprint requests to Ken Resnicow, University of Michigan, School of Public Health, 109 Observatory Street, Room 3867 SPH I, Ann Arbor, MI 48109-2029, USA. E-mail: kresnic@umich.edu An Appendix is available online in the table of contents for this issue: http://journals.cambridge.org/jid_BCP 
and ambivalent clients (Hettema, Steele and Miller, 2005; Lundahl, Kunz, Brownell, Tollefson and Burke, 2010). An effective MI practitioner is able to strategically balance the need to "comfort the afflicted" and "afflict the comfortable"; to balance the expression of empathy with the need to build sufficient discrepancy and urgency to stimulate change.

The essence of MI lies in its spirit; however, specific techniques help ensure such spirit is evoked. One core MI strategy is reflective listening. Reflective listening can be conceptualized as a form of hypothesis testing. The hypothesis can be stated in generic terms as "If I heard you correctly, this is what I think you are saying ..." or "Given what you said, you might feel xxx..." Reflections, particularly by counselors who are new to the technique, often begin with the phrase, "It sounds like..." More skilled counselors often phrase their reflections in a truncated form, such as "You are having trouble with ...", leaving off the assumed "It sounds like..." The goals of reflecting include demonstrating that the counselor has heard and is trying to understand the client, affirming the client's thoughts and feelings without passing judgment, and helping the client resolve ambivalence or resistance and find their own reasons for change.

Once resistance or ambivalence are resolved and motivation is solidified, many practitioners struggle with how best to transition the discussion toward action planning, while still retaining the spirit and style of client centeredness; i.e. moving from the WHY phase to the HOW phase of counseling in a style that is MI-consistent. For many, there is a perception that the counseling style, skills, and strategies used to build motivation are distinct from those used in the action planning phase. The WHY to HOW transition does not, however, necessitate abandoning a client-centered style for a more overtly educational or directive style. Goal setting, action planning, provision of advice, and relapse prevention can be implemented from an autonomy supportive, MI consistent framework. To this end, this article will present a new class of reflection, which we have termed "action reflections", that can be used to help bridge the WHY-HOW gap. Action reflections allow the clinician to maintain a tone and orientation that are consistent with MI, i.e. autonomy support; guiding versus directing, during the action phase of counseling.

\section{Change talk and action reflections}

A core principle of MI is that individuals are more likely to accept and act upon opinions that they voice themselves (Bem, 1972). Therefore, clients are encouraged to express their own reasons and plans for change, which in MI is referred to as change talk. Expression of change talk, particularly a strong crescendo within and across sessions, appears to be a good predictor of future change, and a key mediator of the MI process (Amrhein, Miller, Yahne, Palmer and Fulcher, 2003; Miller and Rose, 2009). Change talk can include clients expressing how they used to feel about themselves when they did " $x$ " behavior in the past or how they might feel in the future if they initiated " $x$ " behavior. The various types of change talk that can be expressed by a client have been conceptualized with the DARN-CAT continuum, where D connotes talking about Desire for change, A connotes Ability for change, Reasons for change, Need for change, Commitment, Activation, and Taking Steps (Rollnick, Miller and Butler, 2008). Intensity of commitment is seen as increasing across the DARN-CAT continuum, with Desire the weakest expression and Taking Steps the strongest expression of change. Thus, action reflections can be conceived as selectively reflecting the A (Activation) and T (Taking Steps) levels of the DARN-CAT continuum. We therefore differentiate between DARN-C and AT types of change talk. The action reflection looks forward rather than inward or backward 
(Apodaca and Longabaugh, 2009; Glynn and Moyers, 2010; Miller and Rose, 2009; Moyers, Martin, Houck, Christopher and Tonigan, 2009). They differ from reflecting change talk as they focus not on the WHY of change, but the HOW, WHEN, or WHERE.

Action Reflections (ARs) also differ from the more common type of reflections such as those that focus on client feelings, rolling with resistance, or acknowledging ambivalence, as ARs usually contain a potential concrete step that the client has directly or obliquely mentioned. By elucidating a specific class of reflection that exclusively addresses what the client can do as opposed to why they might do it, we have found that trainees are better able to invoke the strategy in their clinical practice. Naming it and dedicating time to its conceptualization and practice within training workshops and supervision elevates its visibility and importance and thereby crystallizes its role. It heightens trainees' ability to filter out this type of "change talk"; to reinforce and elicit it with confidence. In sum, because action reflections have a unique clinical objective, address a specific trainee concern, and differ in form and function from other types of reflections, we propose they merit "independent" status in the pantheon of reflections. One research and practice implication of "independent" status for action reflections might be to include this type of reflection as a separate domain in MI fidelity rating systems such as the MITI (Glynn and Moyers, 2010; Moyers, Martin, Manuel, Hendrickson and Miller, 2005; Moyers, Miller and Hendrickson, 2005).

Because the client directly mentioned or alluded to the possible course(s) of action contained within the action reflection or they flow logically from client statements, this type of reflection should not be confused with unsolicited advice. Like any type of reflection, ARs represent the clinician's best guess for what the client has said or, more apropos here, where the conversation might be heading.

The AR can be differentiated from "advice with permission". Advice with permission is a useful, autonomy-supportive means to provide a suggestion NOT mentioned by the client; to provide ideas based on the clinician's experience and intuition. Because the action reflection is usually driven by something the client has previously said or implied, rather than from the clinician's experience or insight, we do not generally find it necessary that permission be explicitly sought. However, the AR can be used as an alternative to "advice with permission" to introduce new ideas and strategies not directly mentioned by the client (see examples 5-7 below). Furthermore, because most action reflections occur during the later phases of MI encounters, practitioner-client rapport should have been already well established, further reducing the need to ask permission. Nonetheless, because any client perception that the counselor is "pushing advice" can elicit resistance and threaten rapport, clinicians are encouraged to invoke the permission strategy when they feel the action reflection might elicit reactance, and the two can be used congruently, for example, permission can precede or be embedded into the action reflection (Brehm and Brehm, 1981; Dillard and Shen, 2005). Given there is a fine line between practitioner advice and the action reflection, clinicians are encouraged to use their clinical judgment as to when to employ either approach.

\section{How to construct ARs}

The tone of the AR reflection is particularly important, with the counselor generally "understating" the potential action step, providing options, and supporting autonomy, using language such as:

1. "You seem to be thinking that you might be able to do X or Y." 
2. "If there was a way to do $\mathrm{X}$ without experiencing barrier $\mathrm{Y}$, then you might be able to move forward..."

3. "You have mentioned several ideas for how to go about this, including..."

4. "There are a range of options you have touched on."

5. "Given what you have said, you might want to consider X or Y."

6. "Given this, it might be helpful to X."

7. "I'm not sure, but X could be an option for you."

Understating the action reduces the likelihood of inducing reactance and allows the client to own the change plan; to inject their energy, enthusiasm and commitment. Ideally, action reflections include multiple choices to support client autonomy. For example; "based on what you said there seems to be several possible options such as X and Y'. There are three subtypes of action reflections, and examples of each can also be found in the online Appendix.

\section{Type 1: Behavior suggestion}

The Behavioral AR can take several forms. The simplest, and often the default type, is to invert client barriers into an action statement, such as "Sounds like, in order to move forward, you might want to address barriers a, b, and c." or "So if we could help reduce the impact of barrier $\mathrm{a}, \mathrm{b}$, or c, your willingness or ability to attempt change might be stronger". Specifically, in the case of smoking cessation, for a client who expressed fears about how to handle craving, this could entail, "So addressing the cravings you experienced last time might help make quitting easier or might give you more confidence to try and quit." Or, for a weight control client who likes eating sweets in the morning; "Finding something in the morning that satisfies your sweet tooth but is a better choice than a donut might be useful."

Sometimes, an action can be presented in a non-specific way as more an umbrella strategy, with the intent of having the client fill in the details. For example, "So you are considering doing something like $x, y$, or z." Specifically, for smoking cessation counseling this could entail, "So medication or a behavioral tip to help reduce cravings might help make quitting easier." For obesity counseling, the reflection might be, "So something sweet like fruit or slightly sweetened cereal might help satisfy your sweet tooth in the morning." Importantly, in both cases the client would have at least mentioned previously some desire to find a way to handle withdrawal if they were to quit smoking, or in the case of the overweight patient, they would have mentioned that they enjoy having sweet vs. savory food in the morning and that they like fruit and/or cereal. Finally, sometimes, based on prior discussion, there is a clear specific solution (or multiple solutions) that the client has mentioned that well match their needs. In these cases, a more specified reflection may be effective. Specifically, this could entail, "So a medicine like nicotine replacement such as Zyban@e, or Chantix@ that helps reduce cravings might help make quitting easier." For a weight control patient who has a morning sweet tooth but also likes fruit, "Given you like bananas and yogurt, that might be an option for you instead of a chocolate croissant in the morning." Again these specifics would generally have been directly or indirectly mentioned by the client, although clinicians should feel free to offer "tailored" advice via the action reflection when they feel the suggestion represents a good fit for their client's needs and flows from their needs and preferences. Whether using the AR or advice with permission, counselors are encouraged to provide multiple options to support client autonomy and provide choice. 


\section{Type 2: Behavior exclusion}

Reflecting back to the client that, given what they have said, there are certain action steps that they might not want to consider can be a highly efficient means to focus the discussion and arrive at an action plan. We call this type of AR an exclusion reflection. For example, if an otherwise happily married woman states that she needs an exercise partner, but she has no one to exercise with, the counselor could reflect back "So, from what you've said, I suspect your husband isn't the answer." Another generic variation might include, "So I might be wrong, but it sounds as though you've thought about trying X solution/option but that doesn't work for or appeal to you." By crossing solutions off the list not only do we communicate to the client that we are listening but it helps avoid the "yes, but" reaction to advice they likely would have rejected.

\section{Type 3: Cognitive suggestion}

Whereas the reflections above focus on behavioral actions, sometimes moving forward can entail modifying cognitions, that is, how the client thinks about the current behavior or possible courses of action. This technique allows the implementation of cognitive therapy strategies within an MI framework. These reflections can be similar to cognitive restructuring techniques, although the new or alternative cognition is presented in the form of a reflection rather than directive advice or education. For example, consider a patient who has approached their prior diet or substance use change attempts from an all or nothing perspective, or despite small successes, does not take credit for the modest changes that have been made, the cognitive reflection might generically take the form of, "Sounds like in order to move forward, thinking about $x$ differently might help." Common cognitive changes can include not applying all-or-nothing thinking, making peace with the lack of immediate benefit or even short-term discomfort, making peace with the chronic nature or severity of the disease, and understanding from prior experiences that they may be able to endure the discomfort in the initial change phase, or that they have been successful in similar prior situations (sometimes also referred to as pulling success forward). Specifically, in the case of a client who failed in prior attempts in part because they have approached behavior change as an all or nothing effort (sometimes called abstinence violation syndrome), the cognitive AR could entail: "Addressing this as an all or nothing thing has not worked for you, and perhaps thinking about this as a more gradual change may be helpful." In the case of a client expressing low efficacy over their ability to change, but who had some success in prior attempts, the reflection might include something like "Accepting the fact that you have dealt with similar discomfort in the past might help make quitting feel a bit less intimidating."

Suggesting new ways of thinking could also include reflections that help the client accept that even small changes can be viewed as success (e.g. cutting down on drinking or smoking is a significant step forward even if the behavior has not been completely eliminated), or using the example of physical activity, that incidental activity still "counts" toward one's physical activity. For example, for a client who feels they are completely sedentary because they no longer go to the gym like they used to in the past but still walks, gardens, plays with her children, "It might be useful for you to consider all your activity when you calculate your daily goals" or "You seem to only include your time in the gym as physical activity but not your walking to and from work or your gardening." Another variant is helping the client view 
their efforts, even if not resulting in success, as a positive expression of commitment rather than a failure.

\section{Client response to action reflections}

Particularly for the latter three types of action reflections, there is the potential for the client to refute the suggestion, even if it is derived from their prior statements and offered in a tentative, understated tone by the clinician. They may elicit a "yes-but" response, either due to resistance or simply because the option does not work for them based on intuition or experience. It is important to bear in mind that, like any other type of reflection, action reflections represent the clinician's best guess for what the client said or where the story is going. They are hypotheses and the productive "foul tip" rule applies here as well. That is, action reflections that are rejected by the client can still yield productive information about what does and does not work or what should or should not be pursued, which helps move the conversation toward resolution. As noted earlier, one way to minimize outright rejection of an action reflection is to provide multiple options within the reflection. For example, "Based on what you have said, it appears you have a few options available...", or in the shortened form "Maybe x or y, might be helpful here". Choice reduces reactance (Brehm and Brehm, 1981; Dillard and Shen, 2005).

In the Appendix, we provide several examples of client statements and potential action reflections. The last example demonstrates an Exclusion Reflection. Note that although we only provide the action reflection for each case, other reflections such as rolling with resistance or acknowledging the "desire" component of change talk could also be evoked by these cases.

\section{Conclusion}

Motivational interviewing has been conceptualized as a prelude to more action oriented therapies (Westra, Arkowitz and Dozois, 2009). This dualism often necessitates that clinicians "code switch" between counseling styles as they progress from the WHY to HOW phases, which can leave practitioners with a fractured clinical experience. The emerging four-process model of MI (i.e. engage, guide, evoke, and plan) recently proposed by Miller and Rollnick (2010), provides practitioners with a unifying framework that delineates discrete styles and strategies that can be used across the full gamut of behavior change counseling. Action reflections represent a key potential strategy for the evoking and planning phases that can enable practitioners to continue functioning from a client-centered, non-directive orientation throughout their encounters.

\section{References}

Amrhein, P. C., Miller, W. R., Yahne, C. E., Palmer, M. and Fulcher, L. (2003). Client commitment language during motivational interviewing predicts drug use outcomes. Journal of Consulting and Clinical Psychology, 71, 862-878.

Apodaca, T. R. and Longabaugh, R. (2009). Mechanisms of change in motivational interviewing: a review and preliminary evaluation of the evidence. Addiction, 104, 705-715.

Bem, D. (1972). Self-perception theory. In L. Berkowitz (Ed.), Advances in Experimental Social Psychology (Vol. 6, pp. 1-62). New York: Academic Press. 
Brehm, S. S. and Brehm, J. W. (1981). Psychological Reactance: a theory of freedom and control. New York: Academic Press.

Dillard, J. P. and Shen, L. (2005). On the nature of reactance and its role in persuasive health communication. Communication Monographs, 72, 144-168.

Glynn, L. H. and Moyers, T. B. (2010). Chasing change talk: the clinician's role in evoking client language about change. Journal of Substance Abuse Treatment, 39, 65-70. doi: S07405472(10)00073-5

Hettema, J., Steele, J. and Miller, W. R. (2005). Motivational Interviewing. Annual Review of Clinical Psychology, 1, 91-111. doi:10.1146/annurev.clinpsy.1.102803.143833

Lundahl, B. W., Kunz, C., Brownell, C., Tollefson, D. and Burke, B. L. (2010). A meta-analysis of Motivational Interviewing: twenty-five years of empirical studies. Research on Social Work Practice, 20, 137-160. doi: 10.1177/1049731509347850

Miller, W. and Rollnick, S. (2010). What's New in MI? Paper presented at the ICMI 2: Second International Conference on Motivational Interviewing, Stockholm, Sweden.

Miller, W. and Rose, G. S. (2009). Toward a theory of motivational interviewing. American Psychologist, 64, 527-537.

Moyers, T. B., Martin, T., Houck, J. M., Christopher, P. J. and Tonigan, J. S. (2009). From in-session behaviors to drinking outcomes: a causal chain for motivational interviewing. Journal of Consulting Clinical Psychology, 77, 1113-1124.

Moyers, T. B., Martin, T., Manuel, J. K., Hendrickson, S. M. and Miller, W. R. (2005). Assessing competence in the use of motivational interviewing. Journal of Substance Abuse Treatment, 28, 1926.

Moyers, T. B., Miller, W. R. and Hendrickson, S. M. (2005). How does motivational interviewing work? Therapist interpersonal skill predicts client involvement within motivational interviewing sessions. Journal of Consulting and Clinical Psychology, 73, 590-598.

Rollnick, S., Miller, W. and Butler, C. C. (2008). Motivational Interviewing in Health Care: helping patients change behavior. New York: Guilford Press.

Westra, H. A., Arkowitz, H. and Dozois, D. J. (2009). Adding a motivational interviewing pretreatment to cognitive behavioral therapy for generalized anxiety disorder: a preliminary randomized controlled trial. Journal of Anxiety Disorders, 23, 1106-1117. 